Pacific Journal of Mathematics

JESSEN'S THEOREM ON RIEMANN SUMS FOR LOCALLY 


\title{
JESSEN'S THEOREM ON RIEMANN SUMS FOR LOCALLY COMPACT GROUPS
}

\author{
Kenneth A. Ross And Karl Stromberg
}

Throughout this paper $G$ denotes a locally compact group and $\left\{H_{n}\right\}$ denotes an increasing sequence of closed subgroups of $G$ whose union $H$ is dense in $G$. For each $n, \Delta_{n}$ denotes the modular function on $H_{n}$ and $\Delta$ denotes the modular function on $G$. Then $\lim _{n} \Delta_{n}(x)=\Delta(x)$ for each $x \in H$. For each $n, \lambda_{n}$ denotes a left Haar measure on $H_{n}$ and $\lambda$ denotes a left Haar measure on $G$. For a function $f$ on $G$ and an $x$ in $G,{ }_{x} f$ denotes the function ${ }_{x} f(y)=f(x y)$. The main theorem states that if $\Delta_{n}$ is the restriction of $\Delta$ to $H_{n}$ for all sufficiently large $n$, then there is a "normalizing" sequence $\left\{\alpha_{n}\right\}$ of positive numbers such that for every $f$ in $\mathfrak{L}_{1}(G, \lambda)$

$$
\mathrm{m}_{n} \alpha_{n} \int_{H_{n}} f d \lambda_{n}=\int_{\theta} f d \lambda
$$

for $\lambda$-locally almost all $x$ in $G$. The hypotheses regarding the $\Delta n$ 's and $\Delta$ hold in all cases known to the authors. In particular, they hold if the $H_{n}$ 's are unimodular (hence if they are Abelian, compact, or discrete) or if the $H_{n}$ 's are open subgroups or normal subgroups. If $G$ is the compact group [0,1 [ with addition modulo 1 , if the $H_{n}$ 's are the finite groups $\left\{k 2^{-n}: 0 \leqq k \leqq 2^{n}-1\right\}$ with counting measure $\lambda_{n}$, and if $\alpha_{n}=2^{-n}$, then the left side of (1) is a Riemann sum and (1) becomes Jessen's theorem.

Jessen's theorem [10] states that if $f$ is a function on the real line that has period 1 and is Lebesgue summable on $[0,1]$, then

$$
\lim _{m} 2^{-n} \sum_{k=0}^{2^{n}-1} f\left(x+\frac{k}{2^{n}}\right)=\int_{0}^{1} f(y) d y
$$

for almost all $x$ in $[0,1]$. Jessen observed that in proving (2) he actually proved that

$$
\lim _{n} \frac{1}{m_{n}} \sum_{k=0}^{m_{n}-1} f\left(x+\frac{k}{m_{n}}\right)=\int_{0}^{1} f(y) d y \quad \text { a.e. }
$$

for any sequence $\left\{m_{n}\right\}$ of positive integers where $m_{n} \mid m_{n+1}$ for all $n$. Since such sequences $\left\{m_{n}\right\}$ correspond to all possible increasing sequences of closed subgroups of [0,1[, the generalization stated in (1) gives no new information about the case $G=[0,1[$.

Relation (3) fails for some functions in $\mathfrak{R}_{1}([0,1[)$ in the case that $m_{n}=n$. This was shown by Marcinkiewicz and Zygmund [12] and 
by Ursell [15]. Rudin [13] showed that there are many sequences $\left\{m_{n}\right\}$ and bounded functions $f$ in $\mathfrak{Q}_{1}([0,1[)$ for which (3) fails and his strong negative theorem emphasizes that the divisibility properties of the $m_{n}$ 's are crucial in Jessen's theorem. These results show that (1) cannot be proved for an arbitrary sequence $\left\{H_{n}\right\}$ whose union is dense. Salem [14] gives a generalization on [0,1[ of Jessen's theorem. Another generalization is given by Civin [3].

Notation and terminology not explicitly defined here can be found in [8] or [9]. The first theorem contains a number of equivalent natural conditions any of which could serve as the definition of a "normalizing sequence". We make a formal definition after the theorem.

THEOREM 1. For a sequence $\left\{\alpha_{n}\right\}$ of positive numbers, the following conditions are equivalent:

(i ) $\lim _{n} \alpha_{n} \int_{H_{n}} f_{0} d \lambda_{n}=\int_{\theta} f_{0} d \lambda$

for some nonzero $f_{0}$ in $\mathfrak{\mho}_{\mathrm{jo}}^{+}(G)$;

(ii) $\lim _{n} \alpha_{n} \int_{\boldsymbol{B}_{n}} f d \lambda_{n}=\int_{G} f d \lambda$

for all $f \in \mathbb{C}_{\mathrm{j}}(G)$;

(iii) $\lim _{n} \alpha_{n} \lambda_{n}\left(U_{0} \cap H_{n}\right)=\lambda\left(U_{0}\right)$

for some nonvoid open set $U_{0}$ in $G$ such that $U_{0}^{-}$is compact and $\lambda\left(\right.$ bdry $\left.U_{0}\right)=0$;

(iv) $\lim _{n} \alpha_{n} \lambda_{n}\left(U \cap H_{n}\right)=\lambda(U)$

for all open sets $U$ such that $U^{-}$is compact and $\lambda($ bdry $U)=0 .{ }^{1}$

Proof. (i) $\Rightarrow$ (ii). There is an $h \in H$ such that $f_{0}(h) \neq 0$. Then ${ }_{n}\left(f_{0}\right)(e) \neq 0$ and (i) is satisfied by ${ }_{h}\left(f_{0}\right)$. We select a sequence $\left\{\beta_{n}\right\}$ such that

$$
\beta_{n} \int_{B_{n}}{ }_{h}\left(f_{0}\right) d \lambda_{n}=\int_{\theta}{ }_{h}\left(f_{0}\right) d \lambda
$$

for all $n$; clearly $\lim _{n} \beta_{n} \alpha_{n}^{-1}=1$. We now use the fact that if $\left\{H_{\gamma}\right\}$ is a net of closed subgroups converging to a closed subgroup $H_{0}$ in the sense of Hausdorff and if the Haar measures $\lambda_{\gamma}$ on $H_{\gamma}$ are normalized so that $\int_{H_{\gamma}} g d \lambda_{\gamma}=\int_{H_{0}} g d \lambda_{0}$ for some $g \in \mathfrak{E}_{\jmath \jmath}^{+}$where $g(e) \neq 0$, then

$$
\lim _{\gamma} \int_{H_{\gamma}} f d \lambda_{\gamma}=\int_{H_{0}} f d \lambda_{0}
$$

for all $f \in \mathbb{C}_{00}$. This is due to J. M. G. Fell; see the appendix to [7]; the proof uses an earlier result of Fell [5]. This fact is also proved by Bourbaki [1] (in §5) and by Flachsmeyer and Zieschang [6]

\footnotetext{
1 Such sets $U$ are sometimes called "continuity sets for $\lambda$ ".
} 
(Satz 1). ${ }^{2}$ Clearly $G$ is the limit of the sequence $\left\{H_{n}\right\}$ in the sense of Hausdorff and relation (1) is a special case of (2). Therefore

$$
\lim _{n} \beta_{n} \int_{H_{n}} f d \lambda_{n}=\int_{\theta} f d \lambda
$$

for all $f \in \mathfrak{C}_{00}$. Assertion (ii) follows from this since $\lim _{n} \beta_{n} \alpha_{n}^{-1}=1$.

(ii) $\Rightarrow$ (iv). Let $U$ be an open set such that $U^{-}$is compact and $\lambda($ bdry $U)=0$. If $f \in \mathfrak{\Im}_{00}$ and $f \geqq \xi_{U^{-}}$, then

$$
\begin{aligned}
\lim \sup _{n} \alpha_{n} \lambda_{n}\left(U \cap H_{n}\right) & \leqq \lim _{n} \alpha_{n} \int_{H_{n}} f d \lambda_{n} \\
& =\int_{\theta} f d \lambda .
\end{aligned}
$$

Since

$$
\lambda(U)=\lambda\left(U^{-}\right)=\inf \left\{\int_{G} f d \lambda: f \in \mathfrak{\Xi}_{00}, f \geqq \xi_{U^{-}}\right\},
$$

we obtain

$$
\lim \sup _{n} \alpha_{n} \lambda_{n}\left(U \cap H_{n}\right) \leqq \lambda(U) .
$$

A similar argument using

$$
\lambda(U)=\sup \left\{\int_{\theta} f d \lambda: f \in \mathfrak{夭}_{00}, f \leqq \xi_{\sigma}\right\}
$$

shows that

$$
\lambda(U) \leqq \liminf _{n} \alpha_{n} \lambda_{n}\left(U \cap H_{n}\right) .
$$

(iv) $\Rightarrow$ (iii) is obvious.

(iii) $\Rightarrow$ (i). Let $f_{0}$ be any nonzero function in $\mathfrak{c}_{00}^{+}$. Clearly there is a sequence $\left\{\beta_{n}\right\}$ of positive numbers for which

$$
\lim _{n} \beta_{n} \int_{\boldsymbol{B}_{n}} f_{0} d \lambda_{n}=\int_{\theta} f_{0} d \lambda .
$$

The already proved implication (i) $\Rightarrow$ (iv) applied to $\left\{\beta_{n}\right\}$ yields

$$
\lim _{n} \beta_{n} \lambda_{n}\left(U_{0} \cap H_{n}\right)=\lambda\left(U_{0}\right) .
$$

By supposition

2 Yet another proof, which uses the Hahn-Banach theorem, can be given for this result. There are at least two other proofs for the compact case. One uses the fact that the semigroup of probability measures on $G$ is compact in the weak-* topology and the other uses the fact that $\lim _{n} \alpha_{n} \hat{\lambda}_{n}=\hat{\lambda}$ pointwise where $\hat{\lambda}_{n}$ and $\hat{\lambda}$ are the Fourier-Stieltjes transforms on the space of equivalence classes of irreducible unitary representations of $G$. 


$$
\lim _{n} \alpha_{n} \lambda_{n}\left(U_{0} \cap H_{n}\right)=\lambda\left(U_{0}\right)
$$

and it follows that $\lim _{n} \alpha_{n} \beta_{n}^{-1}=1$. This equality and (3) imply that (i) holds for $\left\{\alpha_{n}\right\}$ and $f_{0}$.

As noted in the proof of Theorem 1 , sequences $\left\{\alpha_{n}\right\}$ satisfying (i) always exist trivially. If $G$ is compact and if $\lambda_{n}\left(H_{n}\right)=\lambda(G)$ for all $n$, then all the conditions of Theorem 1 hold for the sequence $\alpha_{n}=1$.

DeFinition. A sequence $\left\{\alpha_{n}\right\}$ satisfying the equivalent conditions of Theorem 1 is called a normalizing sequence for the family $\left\{\lambda, \lambda_{1}, \lambda_{2}, \cdots\right\}$ of left Haar measures.

It is easy to prove that if $\left\{\alpha_{n}\right\}$ is a normalizing sequence, then another sequence $\left\{\beta_{n}\right\}$ of positive numbers is a normalizing sequence if and only if $\lim _{n} \alpha_{n} \beta_{n}^{-1}=1$.

The next two theorems tell us more about normalizing sequences.

LeMma 1. If $\left\{\alpha_{n}\right\}$ is a normalizing sequence and if $F$ is a compact subset of $G$, then there is a finite constant $c_{F}$, depending only upon $F$, such that

(i) $\alpha_{n} \lambda_{n}\left(x F \cap H_{n}\right) \leqq c_{F}$

for all $x \in G$ and all $n$. The constant $c_{F^{r}}$ can be taken to be $\sup _{n} \alpha_{n} \lambda_{n}\left(F^{-1} F \cap H_{n}\right) .^{3}$

Proof. Choose $g$ in $\mathfrak{\complement}_{00}^{+}$such that $g \geqq \xi_{F^{-1} F}$; then

$$
c_{F}=\sup _{n} \alpha_{n} \lambda_{n}\left(F^{-1} F \cap H_{n}\right) \leqq \sup _{n} \alpha_{n} \int_{H_{n}} g d \lambda_{n}<\infty .
$$

Consider any $x$ and $n$. If $x F \cap H_{n}=\varnothing$, then (i) is plain. Otherwise $x a=h$ for some $a \in F$ and $h \in H_{n}$. Then

$$
\begin{aligned}
\alpha_{n} \lambda_{n}\left(x F \cap H_{n}\right) & =\alpha_{n} \int_{H_{n}} \xi_{x F} d \lambda_{n} \\
& =\alpha_{n} \int_{H_{n} h^{-1}}\left(\xi_{a-1}\right) d \lambda_{n} \\
& =\alpha_{n} \int_{H_{n}} \xi_{a^{-1} F} d \lambda_{n} \leqq \alpha_{n} \int_{H_{n}} \xi_{F^{-1} F} d \lambda_{n} \leqq c_{F} .
\end{aligned}
$$

Notation. For the remainder of the paper, whenever $F$ is a compact subset of $G, c_{F}$ will denote the constant in Lemma 1 . If $G$ is compact and $\lambda_{n}\left(H_{n}\right)=\lambda(G)=1$ for all $n$, then we take $\alpha_{n}=1$ for all $n$ and $c_{F}=1$ for all $F$.

3 The existence of $c_{F}$ can also be deduced from the proof of Fell's theorem [7] or from Hilfssatz 1 of [6]. 
THEOREM 2. Let $\left\{\alpha_{n}\right\}$ be a normalizing sequence. If $f \in \mathfrak{E}_{00}(G)$, then

(i) $\lim _{n} \alpha_{n} \int_{\mathbb{H}_{n}} f d \lambda_{n}=\int_{G} f d \lambda$

and

(ii) $\lim _{n} \alpha_{n} \int_{H_{n}} f_{x} d \lambda_{n}=\Delta\left(x^{-1}\right) \int_{\theta} f d \lambda$

uniformly on compact subsets of $G$.

Proof. ${ }^{4}$ The pointwise convergence of (i) and (ii) follows from Theorem 1. Let $F$ be any compact subset of $G$. An Ascoli theorem (Theorem 15, page 232 of [11]) states that pointwise convergence implies uniform convergence on compact sets provided that the functions involved belong to an equicontinuous family of functions. Thus it suffices to prove that the family of functions consisting of all

$$
\dot{\phi}_{n}(x)=\alpha_{n} \int_{\pi_{n}} f d \lambda_{n} \text { and all } \psi_{n}(x)=\alpha_{n} \int_{H_{n}} f_{x} d \lambda_{n}
$$

is equicontinuous on $F$. Let $E$ be a compact set containing the support of $f$. Let $c=c_{E \cup E F^{-1}}$; by Lemma 1 ,

$$
\alpha_{n} \lambda_{n}\left(x\left(E \cup E F^{-1}\right) \cap H_{n}\right) \leqq c
$$

for all $x$ and $n$. Given $\varepsilon>0$, select a neighborhood $V$ of the identity $e$ such that $x y^{-1} \in V$ implies $\left\|{ }_{x} f-{ }_{y} f\right\|_{u}<\varepsilon / 2 c$ and $\left\|f_{x}-f_{y}\right\|_{u}<\varepsilon / c$. Then $x y^{-1} \in V$ implies

$$
\begin{aligned}
\left|\dot{\phi}_{n}(x)-\dot{\phi}_{n}(y)\right| & \leqq \alpha_{n} \int_{H_{n}}\left|{ }_{x} f-{ }_{y} f\right| d \lambda_{n} \\
& =\alpha_{n} \int_{H_{n}}\left|{ }_{x} f-{ }_{y} f\right| \xi_{x^{-1} E \cup y^{-1} E} d \lambda_{n} \\
& \leqq \frac{\varepsilon}{2 c} \alpha_{n} \lambda_{n}\left(x^{-1} E \cap H_{n}\right)+\frac{\varepsilon}{2 c} \alpha_{n} \lambda_{n}\left(y^{-1} E \cap H_{n}\right) \leqq \varepsilon ;
\end{aligned}
$$

if, in addition, $x$ and $y$ are in $F$, then

$$
\begin{aligned}
\left|\psi_{n}(x)-\psi_{n}(y)\right| & \leqq \alpha_{n} \int_{H_{n}}\left|f_{x}-f_{y}\right| \xi_{E F^{-1}} d \lambda_{n} \\
& \leqq \frac{\varepsilon}{c} \alpha_{n} \lambda_{n}\left(E F^{-1} \cap H_{n}\right) \leqq \varepsilon
\end{aligned}
$$

THEOREM 3. Let $\left\{\alpha_{n}\right\}$ be a normalizing sequence. Then $G / H_{n}$ is compact for some $n$ if and only if

\footnotetext{
4 The proof for compact $G$ was kindly given us by Thomas Paine.
} 
(i ) $\lim _{n} \alpha_{n} \int_{B_{n}} f d \lambda_{n}=\int_{G} f d \lambda$ uniformly on $G$ for all $f \in \mathfrak{E}_{00}(G)$.

Proof. Let $\phi_{n}(x)=\alpha_{n} \int_{B_{n}} f d \lambda_{n}$.

Suppose that $G / H_{n_{0}}$ is compact for some $n_{0}$. Then there is a compact set $F$ in $G$ such that $F H_{n}=G$ for all $n \geqq n_{0}$; see 5.24.b of [8]. By Theorem 2 there is an $n_{1} \geqq n_{0}$ such that $\left|\phi_{n}(y)-\int_{\theta} f d \lambda\right|<\varepsilon$ for all $y \in F$ and $n \geqq n_{1}$. For any $x$ in $G$ and $n \geqq n_{1}, x=y h$ for some $y \in F$ and $h \in H_{n}$ and hence

$$
\begin{aligned}
\phi_{n}(x) & =\alpha_{n} \int_{\boldsymbol{B}_{n}} f d \lambda_{n}=\alpha_{n} \int_{\boldsymbol{B}_{n}}{ }_{h}\left({ }_{y} f\right) d \lambda_{n} \\
& =\alpha_{n} \int_{\boldsymbol{H}_{n}}{ }_{y} f d \lambda_{n}=\phi_{n}(y)
\end{aligned}
$$

It follows that $\left|\phi_{n}(x)-\int_{G} f d \lambda\right|<\varepsilon$ for all $x \in G$ and $n \geqq n_{1}$.

Suppose now that (i) holds. Let $f$ be a nonzero function in $\mathfrak{F}_{00}^{+}$ and let $F$ be a compact set containing its support. Since $\int_{G} f d \lambda>0$, there is an $n$ such that $\alpha_{n} \int_{B_{n}} f d \lambda_{n}>0$ for all $x \in G$. Then $x \in G$ implies that ${ }_{x} f(h) \neq 0$ for some ${ }^{H_{n}} h \in H_{n}$, hence $x h \in F$ and $x \in F H_{n}^{-1}$. Therefore $G=F H_{n}$ and $G / H_{n}$ is compact.

The next theorem relates the modular function on $G$ to the modular functions on the $H_{n}$ 's.

THEOREM 4. If $F$ is a compact subset of some $H_{m}$, then $\lim _{n} \Delta_{n}(x)=\Delta(x)$ uniformly on $F$. In particular, $\lim _{n} \Delta_{n}(x)=\Delta(x)$ for all $x \in H$.

Proof. Let $\left\{\alpha_{n}\right\}$ be a normalizing sequence and let $f$ be a nonzero function in $\widetilde{F}_{j 3}^{+}$. By Theorem 2, we have

$$
\begin{aligned}
\lim _{n} \Delta_{n}(x) \alpha_{n} \int_{\boldsymbol{H}_{n}} f d \lambda_{n} & =\lim _{n} \alpha_{n} \int_{\boldsymbol{B}_{n}} f_{x^{-1}} d \lambda_{n} \\
& =\Delta(x) \int_{\theta} f d \lambda
\end{aligned}
$$

uniformly on $F$. Since

$$
\lim _{n} \alpha_{n} \int_{\boldsymbol{H}_{n}} f d \lambda_{n}=\int_{\theta} f d \lambda \neq 0
$$

we infer that $\lim _{n} \Delta_{n}(x)=\Delta(x)$ uniformly on $F$. 
Note that $\Delta_{n}=\Delta \mid H_{n}$ whenever $H_{n}$ is a normal subgroup of $G$; see 15.23 of [8]. If $H_{n}$ is not normal, then the identity $\Delta_{n}=\Delta \mid H_{n}$ may fail to hold. It seems unlikely that $\Delta_{n}=\Delta \mid H_{n}$ must hold for sufficiently large $n$, but the authors unfortunately have not been able to produce an example to settle this question. Further comments about this question follow Theorem 5 .

We next prove two lemmas that are needed in order to prove in Theorem 5 our main result, namely, our generalization of Jessen's theorem. The first lemma is a consequence of a result of Edwards and Hewitt [4].

Lemma 2. Suppose that $\left\{\mu_{n}\right\}$ is a sequence of nonnegative Borel measures on $G$. Then, for every $f \in \mathfrak{R}_{1}(G, \lambda),{ }_{x} f$ is $\mu_{n}$-measurable for $\lambda$-locally almost all $x \in G$. Suppose also that

(i) $\lim _{n} \int_{G} f d \mu_{n}=\int_{G} f d \lambda$

for all $f \in \mathfrak{S}_{00}(G)$ and that

(ii) $\sup _{n} \int_{\theta} f d \mu_{n}<\infty \lambda$-locally a.e. for all $f \in \mathcal{R}_{1}^{+}(G, \lambda)$. Then

(iii) $\lim _{n} \int_{G} f d \mu_{n}=\int_{G} f d \lambda \lambda$-locally a.e. for all $f \in \mathfrak{R}_{1}(G, \lambda)$.

Proof. In their Theorem 1.6, Edwards and Hewitt [4] prove the following. Suppose $E$ is a real semimetrizable topological vector space of the second category and that $(S, \mathscr{L}, \mu)$ is a measure space. Let $\Im$ be the family of all $\mathscr{C}$-measurable functions from $S$ into $[0, \infty]$, where any two functions in $\widetilde{F}$ that are equal $\mu$-locally almost everywhere are identified. Suppose $\left\{P_{\alpha}\right\}$ is a countable net of sublinear operators from $E$ into $\mathfrak{F}$ satisfying

(1) for each $\alpha, \lim _{n} f_{n}=f$ in $E$ implies that $\lim _{k} P_{\alpha} f_{n_{k}}=P_{\alpha} f \mu$ locally a.e. for some subsequence $\left\{f_{n_{k}}\right\}$ of $\left\{f_{n}\right\}$,

and

(2) $P f(s)=\sup _{\alpha} P_{\alpha} f(s)$ is finite $\mu$-locally a.e. for every $f \in E$.

Let $E_{0}$ be the set of $f$ in $E$ for which $\lim _{\alpha} P_{\alpha} f(s)=0 \mu$-locally a.e. Then $E_{0}$ is a closed vector subspace of $E$.

It suffices to prove (iii) for $f$ in $\mathfrak{R}_{1}^{r}(G)$. Let $E=\mathfrak{Q}_{1}^{r}$ and, for each positive integer $m$ and $f \in \mathfrak{R}_{1}^{r}$, let

$$
P_{m} f(x)=\left|\int_{G} f d \mu_{m}-\int_{G} f d \lambda\right| \quad \text { for } x \in G .
$$


Suppose that $G$ is $\sigma$-compact. If $h$ is a $\lambda$-null function on $G$, then $|h|$ is dominated by a Borel measurable $\lambda$-null function $k$. Then $(x, y) \rightarrow$ $k(x y)$ is Borel measurable on $G \times G$ and an application of Fubini's theorem (13.9 of [8]) shows that ${ }_{x} k$ is $\mu_{m}$-measurable for $\lambda$-almost all $x$ and that $\int_{\theta_{x}} k d \mu_{n}$ is $\lambda$-null. The same remarks thus apply to $h$. A similar application of Fubini's theorem shows that $\int_{a} f d \mu_{m}$ is $\lambda$ measurable for any $f \in \mathfrak{R}_{1}(G, \lambda)$. Therefore $P_{m} f$ is $\lambda$-measurable and is well-defined in the sense that if $f=g \lambda$-a.e., then $P_{m} f=P_{m} g$ in $\mathfrak{F}$. If $G$ is not $\sigma$-compact, the same statement can be proved by making a similar argument on its open $\sigma$-compact subgroups. It is easy to see that each $P_{m}$ is sublinear:

$$
P_{m}(\alpha f)=|\alpha| P_{m}(f) \text { and } P_{m}(f+g) \leqq P_{m} f+P_{m} g
$$

$\lambda$-locally a.e., where $\alpha$ is real.

To prove (1), fix $m$ and suppose that $\lim _{n}\left\|f_{n}-f\right\|_{1}=0$ where $f$ and each $f_{n}$ belong to $\mathfrak{R}_{1}^{r}$. Since $\lim _{n} \int_{G} f_{n} d \lambda=\int_{G} f d \lambda$, it suffices to prove that the sequence

$$
s_{n}(x)=\left|\int_{\theta}{ }_{x}\left(f_{n}\right) d \mu_{m}-\int_{\theta} f d \mu_{m}\right|
$$

of functions has a subsequence that converges $\lambda$-locally a.e. to 0 . For positive integers $k$, choose $n_{k}$ so that $\left\|f_{n_{k}}-f\right\|_{1}<4^{-k}$ and let $g=\sum_{k=1}^{\infty} 2^{k}\left|f_{n_{k}}-f\right|_{\text {. Then }} g$ belongs to $\Omega_{1}$ and $\int_{G} g d \mu_{m}$ exists and is finite $\lambda$-locally a.e. For any $x$ such that $\int_{\theta}{ }_{x} g d \mu_{m}<\infty$, we have

$$
s_{n_{k}}(x)=\left|\int_{\theta}{ }_{x}\left(f_{n_{l_{b}}}-f\right) d \mu_{m}\right| \leqq 2^{-k} \int_{G}{ }_{x} g d \mu_{m}
$$

and hence $\lim _{k} s_{n_{k}}(x)=0$. This proves (1), and (2) follows immediately from (ii).

Let $E_{0}$ consist of all $f$ in $\mathbb{R}_{1}^{r}$ such that $\lim _{m} P_{m} f(x)=0 \lambda$-locally a.e. Equivalently $E_{0}$ consists of the functions in $\Re_{1}^{r}$ for which (iii) holds and so we need only prove that $E_{0}=\mathbb{R}_{1}^{r}$. For any $f \in \mathbb{C}_{00}^{r}$ and $x \in G$, (i) applied to ${ }_{x} f$ shows that $\lim _{m} P_{m} f(x)=0$. Therefore $\mathfrak{C}_{00}^{r} \subset E_{0}$; the theorem of Edwards and Hewitt asserts that $E_{0}$ is closed in $\mathfrak{Q}^{r}$ and hence $E_{0}=\Omega_{1}^{r}$.

LEMMA 3. Suppose that $\left\{\alpha_{n}\right\}$ is a normalizing sequence and that $\Delta_{n}=\Delta \mid H_{n}$ for all $n$. Let $f$ be a nonnegative Borel measurable function on $G$. Let

$$
f^{*}(x)=\sup _{n} \alpha_{n} \int_{H_{n}} f d \lambda_{n}
$$


for $x \in G$ and for $t \geqq 0$, let $B_{t}^{*}=\left\{x \in G: f^{*}(x)>t\right\}$. Then for $t \geqq 0$ and every compact subset $F$ of $G$, we have

(i) $t \lambda\left(B_{t}^{*} \cap F\right) \leqq c_{F} \int_{B_{t}^{*}} f d \lambda$.

If $f$ is also in $\mathbb{R}_{1}^{+}(G, \lambda)$, then

(ii) $f^{*}(x)<\infty$

for $\lambda$-locally almost all $\rightsquigarrow$ in $G$.

Proof. ${ }^{5} \quad$ Let $\dot{\phi}_{n}(x)=\alpha_{n} \int_{B_{n}} f d \lambda_{n}$. Let $N$ be a fixed positive integer and let $D_{N}=\left\{x \in G: \sup _{1 \leqq n \leqq N} \phi_{n}(x)>t\right\}$. For $n=1,2, \cdots, N$, let $E_{n}=\left\{x \in G: \phi_{n}(x)>t\right\}$ and let $\mathrm{A}_{n}=E_{n} \cap\left(\bigcup_{k=n+1}^{N} E_{k}\right)^{\prime}$; note that $A_{N}=$ $E_{N}$. Note also that $E_{k} H_{n}=E_{k}$ for $n \leqq k$ and hence $A_{n} H_{n}=A_{n}$ for all $n \leqq N$. Recall that $\alpha_{n} \lambda_{n}\left(x F \cap H_{n}\right) \leqq c_{F}$ for all $x$ and $n$ by Lemma 1. For all $n$, we have

$$
\begin{aligned}
t \lambda\left(A_{n} \cap F^{\prime}\right) & \leqq \int_{A_{n} \cap F^{F}} \phi_{n} d \lambda \\
& =\int_{A_{n} \cap F^{F}} \alpha_{n} \int_{H_{n}} f(x y) d \lambda_{n}(y) d \lambda(x) \\
& =\alpha_{n} \int_{H_{n}} \int_{G} \xi_{A_{n} \cap F^{\prime}}(x) f(x y) d \lambda(x) d \lambda_{n}(y) \\
& =\alpha_{n} \int_{H_{n}} \Delta\left(y^{-1}\right) \int_{G} \xi_{\left(A_{n} \cap F^{\prime}\right)}-1(x) f(x) d \lambda(x) d \lambda_{n}(y) \\
& =\int_{G} f(x) \alpha_{n} \int_{H_{n}} \Delta_{n}\left(y^{-1}\right) \xi_{\left(A_{n} \cap F^{-1}\right)}(y) d \lambda_{n}(y) d \lambda(x) \\
& =\int_{G} f(x) \alpha_{n} \int_{H_{n}} \xi_{x^{-1}\left(A_{n} \cap F^{\prime}\right)}(y) d \lambda_{n}(y) d \lambda(x) \\
& =\int_{G} f(x) \alpha_{n} \lambda_{n}\left(x^{-1}\left(A_{n} \cap F\right) \cap H_{n}\right) d \lambda(x) \\
& =\int_{A_{n}} f(x) \alpha_{n} \lambda_{n}\left(x^{-1}\left(A_{n} \cap F\right) \cap H_{n}\right) d \lambda(x) \\
& \leqq c_{F} \int_{A_{n}} f d \lambda .
\end{aligned}
$$

The last equality follows from the fact that $A_{n} H_{n}=A_{n}$. Since $D_{n}=$ $\bigcup_{n=1}^{N} A_{n}$ and the union is disjoint, we infer that $t \lambda\left(D_{N} \cap F\right) \leqq c_{F} \int_{D_{N}} f d \lambda$. Inequality (i) now follows from the fact that $B_{t}^{*}$ is the union of the increasing sequence $\left\{D_{N}\right\}$ of sets.

To prove (ii) we need to show that $B=\bigcap_{t=1}^{\infty} B_{t}^{*}$ is locally null. For a compact set $F$, (i) shows that $t \lambda\left(B_{t}^{*} \cap F\right) \leqq c_{F}\|f\|_{1}$ and hence $\lim _{t \rightarrow \infty} \lambda\left(B_{t}^{*} \cap F\right)=0$. Therefore $\lambda(B \cap F)=0$ and $B$ is locally null.

${ }_{5}$ This proof of (i) is a modification of the proof of one of Jessen's lemmas [10]. 
An example showing the necessity of the hypothesis regarding $\Delta$ and the $\Delta_{n}$ 's will be given after Theorem 5 . In Theorem 6 , we will obtain sharper results about the function $f^{*}$ for the case that $G$ is compact.

THEOREM 5. Suppose that $\left\{\alpha_{n}\right\}$ is a normalizing sequence and that $\Delta_{n}=\Delta \mid H_{n}$ for all sufficiently large $n$. If $f$ is in $\aleph_{1}(G, \lambda)$, then

(i) $\lim _{n} \alpha_{n} \int_{H_{n}} f d \lambda_{n}=\int_{G} f d \lambda$ for $\lambda$-locally almost all $x$ in $G$.

Proof. Choose $n_{0}$ so that $\Delta_{n}=\Delta \mid H_{n}$ for $n \geqq n_{0}$. We apply Lemma 2 to the sequence $\left\{\alpha_{n} \lambda_{n}: n \geqq n_{0}\right\}$ of measures; these measures may, of course, be regarded as defined on $G$. Hypothesis (i) follows from Theorem 1. To prove (ii), we replace $f$ by a Borel measurable function that is equal to it $\lambda$-a.e. and then apply (ii) of Lemma 3.

REMARKS AND ExAMPLES. The hypotheses regarding $\Delta$ and the $\Delta_{n}$ 's in Lemma 3 and Theorem 5 are there because Lemma 3 is false otherwise and because we are unable to prove or disprove Theorem 5 without this hypothesis; compare with our remarks following Theorem 4. We now give an example to show that (ii) of Lemma 3 can fail if $\Delta_{1} \neq \Delta \mid H_{1}$. Let $G$ be the group of real matrices $\left(\begin{array}{ll}x & y \\ 0 & z\end{array}\right), x>0$, $z>0$; we abbreviate $\left(\begin{array}{ll}x & y \\ 0 & z\end{array}\right)$ as $(x, y, z)$. See 15.28.b of $[8]$. Let $H_{1}=\{(x, 0,1): x>0\}$ and for $n \geqq 2$ let

$$
H_{n}=\left\{\left(x, y, \exp \left(k \cdot 2^{-n}\right)\right): x>0, y \in R, k \in Z\right\} .
$$

The characteristic function $f$ of $\{(x, y, z): x>1,|y|<1,1<z<e\}$ is in $\mathfrak{\Omega}_{1}(G)$; its left Haar integral is $\int_{1}^{\infty} \int_{-1}^{1} \int_{1}^{e} x^{-2} z^{-1} d z d y d x=2$. If $(a, b, c) \in G$, then $\int_{H_{1}(a, b, c)} f d \lambda_{1}$ is the integral over $H_{1}$ of the characteristic function of $\{(x, y, z): a x>1,|a y+b z|<1,1<c z<e\}$. If $a>0,|b|<1$, and $1<c<e$, the intersection of this set with $H_{1}$ is $\left\{(x, 0,1): x>a^{-1}\right\}$ and therefore

$$
\int_{H_{1}}(a, b, c) f d \lambda_{1}=\int_{a^{-1}}^{\infty} d \lambda_{1}=\infty
$$

Thus $f^{*}(a, b, c)=\infty$ on the open set $\{(a, b, c): a>0,|b|<1,1<c<e\}$ which is certainly not $\lambda$-locally null.

If one applies Theorem 5 to the real line $R$ and its subgroups $H_{n}=\left\{k 2^{-n}: k \in Z\right\}$, one finds that

$$
\lim _{n} 2^{-n} \sum_{k=-\infty}^{\infty} f\left(x+\frac{k}{2^{n}}\right)=\int_{-\infty}^{\infty} f(y) d y \text { a.e. }
$$


whenever $f$ is in $\mathbb{R}_{1}(R)$.

Groups $G$ admitting nontrivial increasing sequences $\left\{H_{n}\right\}$ with dense union exist in profusion. For a compact Abelian group, this property holds if and only if the character group $X$ contains a nontrivial decreasing sequence of subgroups whose only common element is the identity. Any nontorsion $X$ has this property as does any $X$ that is a sum or product of an infinite number of subgroups. Some groups without this property are finite products of $Z\left(p^{\infty}\right)$ groups. Thus finite products of the groups $\Delta_{p}$ of $p$-adic integers do not have nontrivial increasing sequences $\left\{H_{n}\right\}$. An allied question asks what groups contain increasing sequences of finite subgroups. No compact infinite Abelian torsion-free group enjoys this property. If $G$ is a direct product of finite groups or groups with this property and there are at most $c$ factors, then $G$ also has this property. Thus $\{-1,1\}^{N_{0}}$ and $T^{c}$ have this property. Finally, of course, there are nonabelian groups that contain increasing sequences of finite subgroups having dense union. Such an example is the group $\subseteq(2)$ of orthogonal transformations of the plane and its subgroups $H_{n}$ of symmetries of the regular polygon with $2^{n}$ sides.

All the results of this paper are simple and uninteresting (though true) when the subgroups $H_{n}$ are open. A locally compact Abelian group is the union of an increasing sequence of proper closed (respectively, open) subgroups if and only if it is not compactly generated; see Lemma 3.3 of [2].

The next technical lemma is needed for our last theorem.

Lemma 4. Let $G$ be a compact group. Let $f$ be a nonnegative Borel measurable function on $G$ and let $f^{*}$ and $B_{t}^{*}$ be as in Lemma 3 . For $u \geqq 0$, let $B_{u}=\{x \in G: f(x)>u\}$. If $0<\alpha<1$ and $t \geqq 0$, then

(i) $(1-\alpha) t \lambda\left(B_{t}^{*}\right) \leqq \int_{B_{\alpha t}} f d \lambda$.

Proof. Let $g=f \xi_{B_{\alpha t}}$; then we have

$$
\begin{aligned}
f^{*}(x) \leqq & \sup _{n} \int_{B_{n}} f(x y) \xi_{B_{\alpha t}}(x y) d \lambda_{n}(y) \\
& +\sup _{n} \int_{B_{n}} f(x y) \xi_{B_{\alpha t}^{\prime}}(x y) d \lambda_{n}(y) \\
\leqq & g^{*}(x)+\alpha t .
\end{aligned}
$$

Thus, letting $C_{u}^{*}=\left\{x \in G: g^{*}(x)>u\right\}$, we have

$$
B_{t}^{*} \subset C_{(1-\alpha) t}^{*} .
$$

Applying (i) of Lemma 3 to $g$ yields 


$$
\begin{aligned}
(1-\alpha) t \lambda\left(B_{t}^{*}\right) & \leqq(1-\alpha) t \lambda\left(C_{(1-\alpha) t}^{*}\right) \\
& \leqq \int_{O_{(1-\alpha) t}^{*}} g d \lambda \leqq \int_{\theta} g d \lambda=\int_{B_{\alpha t}} f d \lambda .
\end{aligned}
$$

THEOREM 6. Let $G$ be a compact group. Let $f$ be a nonnegative Borel measurable function on $G$ and let $f^{*}(x)=\sup _{n} \int_{\boldsymbol{B}_{n}} f d \lambda_{n}$. If $f$ is in $\mathfrak{R}_{p}$ where $1<p<\infty$, then so is $f^{*}$ and

(i) $\left\|f^{*}\right\|_{p} \leqq \frac{p}{p-1}\|f\|_{p} \cdot{ }^{6}$

If $f \in \mathbb{R} \log ^{+} \mathfrak{R}$, then $f^{*} \in \mathfrak{R}_{1}$ and for every $\left.\alpha \in\right] 0,1[$, we have

$$
\left\|f^{*}\right\|_{1} \leqq \frac{1}{\alpha}+\frac{1}{1-\alpha} \int_{\theta} f \log ^{+} f d \lambda \text {. }
$$

If $f \in \mathfrak{\Omega}_{1}$, then $f^{*} \in \mathfrak{Q}_{p}$ for all $0<p<1$ and

(iii) $\left\|f^{*}\right\|_{p} \leqq(1-p)^{-1 / p}\|f\|_{1}$.

This theorem is proved using (i) of Lemma 3 and Lemma 4 in exactly the same way that the Hardy-Littlewood maximal theorems (21.76 and 21.80 of [9]) are deduced from Lemmas 21.75 and 21.79 of [9].

Theorem 6 cannot be extended to locally compact noncompact groups as the following examples show. Consider the group $R$ of real numbers and its subgroups $H_{n}=\left\{k 2^{-n}: k \in Z\right\}$. If $f=\xi_{[0,1[}$, then $f^{*}(x)=1$ for all $x \in R$. A more striking example is given by the function $g(x)=(1 / x) \xi_{[1, \infty[}(x)$. Even though $g$ belongs to $\Omega_{p}(R)$ for all $p>1, g^{*}(x)=\infty$ for all $x \in R ; g^{*}$ is not even locally in $\Re_{p}(R)$.

The authors are indebted to Professor Edwin Hewitt for suggesting this problem.

\section{REFERENCES}

1. N. Bourbaki, Eléments de Mathématique, Livre VI: Integration, Chapitre 8: Convolution et representations, Actualités Sci. Indust., No. 1306, Hermann, Paris, 1963. 2. F. W. Carroll, A difference property for polynomials and exponential polynomials on Abelian locally compact groups, Trans. Amer. Math. Soc. 114 (1965), 147-155.

3. P. Civin, Abstract Riemann sums, Pacific J. Math. 5 (1955), 861-868.

4. R. E. Edwards and E. Hewitt, Pointwise limits for sequences of convolution operators, Acta Math. 113 (1965), 181-218.

5. J. M. G. Fell, A Hausdorff topology for the closed subsets of a locally compact nonHausdorff space, Proc. Amer. Math. Soc. 13 (1962), 472-476.

6. J. Flachsmeyer and $\mathrm{H}$. Zieschang, Über die schwache Konvergenz der Haarschen Masse von Untergruppen, Math. Annalen 156 (1964), 1-8.

7. J. G. Glimm, Families of induced representations, Pacific J. Math. 12 (1962), 885-911.

${ }^{6}$ Conclusion (i) for the case $G=[0,1]$ was given by Jessen [10]. 
8. E. Hewitt and K. A. Ross, Abstract Harmonic Analysis, Vol. 1, Springer-Verlag, Heidelberg, 1963.

9. E. Hewitt and K. Stromberg, Real and Abstract Analysis, Springer-Verlag, Heidelberg, 1965.

10. B. Jessen, On the approximation of Lebesgue integrals by Riemann sums, Ann. of Math. 35 (1934), 248-251.

11. J. L. Kelley, General Topology, D. Van Nostrand Co., Inc., New York, 1955.

12. J. Marcinkiewicz and A. Zygmund, Mean values of trigonometrical polynomials, Fund. Math. 28 (1937), 131-166.

13. W. Rudin, An arithmetic property of Riemann sums, Proc. Amer. Math. Soc. 15 (1964), 321-324.

14. R. Salem, Sur les sommes Riemanniennes des fonctions sommables, Mat. Tidsskr. B (1948), 60-62.

15. H. D. Ursell, On the behaviour of a certain sequence of functions derived from a given one, J. London Math. Soc. 12 (1937), 229-232.

Received October 18, 1965. This research was supported by National Science Foundation Grant GP-3927.

UNIVERSITY OF OREGON 



\section{PACIFIC JOURNAL OF MATHEMATICS}

H. SAMELSON

Stanford University

Stanford, California

J. P. JaNs

University of Washington

Seattle, Washington 98105

\section{EDITORS}

J. DugundJI

University of Southern California Los Angeles, California 90007

RICHARD ARENS

University of California

Los Angeles, California 90024

\section{ASSOCIATE EDITORS}

E. F. BECKENBACH

B. H. NEUMANN

F. WOLF

K. YosidA

\section{SUPPORTING INSTITUTIONS}

UNIVERSITY OF BRITISH COLUMBIA

CALIFORNIA INSTITUTE OF TECHNOLOGY

UNIVERSITY OF CALIFORNIA

MONTANA STATE UNIVERSITY

UNIVERSITY OF NEVADA

NEW MEXICO STATE UNIVERSITY

OREGON STATE UNIVERSITY

UNIVERSITY OF OREGON

OSAKA UNIVERSITY

UNIVERSITY OF SOUTHERN CALIFORNIA
STANFORD UNIVERSITY

UNIVERSITY OF TOKYO

UNIVERSITY OF UTAH

WASHINGTON STATE UNIVERSITY

UNIVERSITY OF WASHINGTON

AMERICAN MATHEMATICAL SOCIETY CHEVRON RESEARCH CORPORATION TRW SYSTEMS

NAVAL ORDNANCE TEST STATION 


\section{Pacific Journal of Mathematics}

\section{Vol. 20, No. $1 \quad$ September, 1967}

Leonard Daniel Baumert, Extreme copositive quadratic forms. II ........ 1

Edward Lee Bethel, A note on continuous collections of disjoint

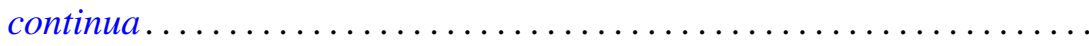

Delmar L. Boyer and Adolf G. Mader, A representation theorem for abelian groups with no elements of infinite p-height ...................

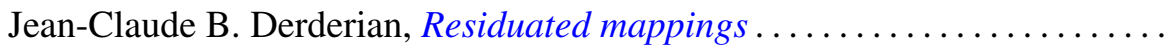

Burton I. Fein, Representations of direct products of finite groups ......... 45

John Brady Garnett, A topological characterization of Gleason parts.......

Herbert Meyer Kamowitz, On operators whose spectrum lies on a circle or

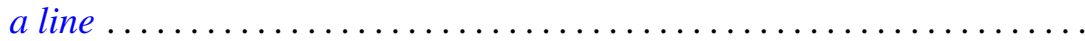

Ignacy I. Kotlarski, On characterizing the gamma and the normal distribution ........................................

Yu-Lee Lee, Topologies with the same class of homeomorphisms ..........

Moshe Mangad, Asymptotic expansions of Fourier transforms and discrete polyharmonic Green's functions ...........................

Jürg Thomas Marti, On integro-differential equations in Banach spaces ....

Walter Philipp, Some metrical theorems in number theory.............. 109

Maxwell Alexander Rosenlicht, Another proof of a theorem on rational

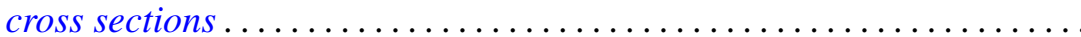

Kenneth Allen Ross and Karl Robert Stromberg, Jessen's theorem on Riemann sums for locally compact groups

Stephen Simons, A theorem on lattice ordered groups, results of Ptak, Namioka and Banach, and a front-ended proof of Lebesgue's theorem...

Morton Lincoln Slater, On the equation $\varphi(x)=\int_{x} x+1 K(\xi) f[\varphi(\xi)] d \xi \ldots$ 155 Arthur William John Stoddart, Existence of optimal controls .. 167 Burnett Roland Toskey, A system of canonical forms for rings on a direct sum of two infinite cyclic groups ....................

Jerry Eugene Vaughan, A modification of Morita's characterization of dimension 